\title{
Percepción de desórdenes musculoesqueléticos y aplicación del método RULA en diferentes sectores productivos: una revisión sistemática de la literatura
}

\author{
Perception of musculoskeletal disorders and RULA method application \\ in different productive sectors: a systematic review of literatura
}

Aanh Eduardo Dimate ${ }^{1}$, Diana Carolina Rodríguez ${ }^{1}$, Anderson Ivan Rocha ${ }^{1}$

Forma de citar: Dimate AE, Rodríguez DC, Rocha AI. Percepción de desórdenes musculoesqueléticos y aplicación del método RULA en diferentes sectores productivos: una revisión sistemática de la literatura. Rev Univ Ind Santander Salud. 2017; 49(1): 57-74. DOI: http://dx.doi.org/10.18273/revsal.v49n1-2017006 @) (i)

\section{RESUMEN}

Introducción: Los desórdenes musculoesqueléticos (DME) constituyen una de las principales causas de enfermedad relacionadas con el trabajo. Numerosos esfuerzos se han realizado a fin de prevenir su aparición; por tanto, múltiples instrumentos de evaluación ergonómica han sido usados. Esta revisión tiene como objetivo identificar la información disponible en bases de datos sobre evaluación del riesgo biomecánico (RULA) y percepción de DME en trabajadores de diversos sectores económicos (Cuestionario Nórdico), para el periodo 2004-2015. Materiales y Métodos: Se realizó una revisión sistemática de los estudios recientes enfocados en la evaluación del nivel de riesgo ergonómico y percepción de DME. Asimismo, fueron tenidas en cuenta las recomendaciones de la declaración PRISMA, tras obtener los artículos en las bases de datos Science direct, Scopus, Pubmed, Springer link, Embase y Ebsco. 13 estudios que cumplieron con los criterios de selección fueron incluidos; se identificaron dos tipos de estudios: de diagnóstico y de intervención. Resultados: Prevalencias altas en espalda alta-baja, cuello, tronco, hombros, mano/ muñeca y rodilla fueron evidenciadas, siendo el género femenino el más afectado. Conclusiones: Los hallazgos sugieren asociación entre índice de masa corporal, edad ( $\geq 25$ años), antigüedad en el trabajo ( $\geq 3$ años), trabajo por destajo, horas de trabajo diario $\geq 8$ horas, número de pacientes atendidos, RULA (nivel 3 y 4 ) y DME en cuello, tronco, brazos, antebrazos, espalda baja y zonas corporales del grupo B.

Palabras clave: Kuorinka (questionnaire nordic), Video terminales (VDTs), Rapid Upper Limb Assesmente (RULA), Desórdenes Musculoesqueléticos (DME), Trabajadores.

\footnotetext{
ABSTRACT

Introduction: Musculoskeletal disorders (MSD) are one of the main causes of work-related diseases; numerous efforts have been made in order to prevent its occurrence. Therefore, multiple ergonomic evaluation instruments have been used. This review aims to identify the available information in databases related to biomechanical risk

1. Fundación Universitaria del Área Andina. Bogotá, Colombia

Correspondencia: Aanh Eduardo Dimate. Dirección: Carrera 14A 71-19. Correo electrónico: aadimate@areandina.edu.co. Teléfono: 3017895125 . 
assessment (RULA) and perception of DME in workers from various economic sectors, period 2004-2015 (Nordic Questionnaire). Materials and Methods: A Systematic review about the recent studies focused on the evaluation of the level of ergonomic risk and perception of DME was performed. The recommendations of the PRISMA statement were taken into account after obtaining the studies from the Science Direct, Scopus, Pubmed, Springer link, Embase and Ebsco databases. 13 studies that complied the selection criteria were included; 2 types of studies were identified: diagnosis and intervention. Results: High prevalence in upper and lower back, neck, trunk, shoulders, hand/wrist, and knee were evidenced, being the female gender the most affected. Conclusions: These findings suggest an association between body mass index, age ( $\geq 25$ years), seniority at work ( $\geq 3$ years), piecework, daily hours of work $\geq 8$ hours, number of patients attended, RULA (level 3 And 4) and DME in the neck, trunk, arms, forearms, lower back and group B body areas.

Keywords: Kuorinka (Nordic questionnaire), Video terminals (VDTs), Rapid Upper Limb Assessment (RULA), Musculoskeletal Disorders (DME), Workers.

\section{INTRODUCCIÓN}

La mayor parte de los DME de origen laboral se van desarrollando con el tiempo y son provocados por el propio trabajo o por el entorno en el que éste se lleva a cabo; las condiciones internas y externas del ser humano inciden en la aparición de estos, aumentando la predisposición de padecerlos por lo que su origen es multifactorial ${ }^{1}$. Estos desordenes incluyen afecciones de los músculos, tendones, vainas tendinosas, síndromes de atrapamiento nervioso, alteraciones articulares y neurovasculares ${ }^{2}$, y por lo general afectan la espalda, cuello, hombros y extremidades superiores e inferiores (con menor frecuencia). Siendo los DME una de las principales causas de absentismo laboral, entrañan por consiguiente un costo considerable para el sistema de salud pública de los países ${ }^{3}$; por ello, se han propuesto modelos mundiales, en los cuales, se busca proteger y promover la salud, seguridad y bienestar de los trabajadores por medio de estrategias dirigidas a mejorar las condiciones laborales ${ }^{4}$.

Los DME de origen laboral constituyen una de las principales causas de enfermedad relacionadas con el trabajo ${ }^{5}$. En Europa, el $24 \%$ de los trabajadores afirmó sufrir dolor de espalda y el 22,8\% se quejó de dolores musculares; específicamente, en Alemania las bajas se estimaron en 24.000 millones de marcos alemanes ${ }^{6}$. Por otro lado, en Estados Unidos (EU) se reportó que las cifras disminuyeron de 435.180 casos en 2003 a 335.390 en 2007, siendo los costos directos de US $\$ 1.5$ mil millones y los indirectos de US $\$ 1,1$ billones para el mismo años ${ }^{7}$. De esta forma, se confirma según la NIOSH, que el costo asociado con los DME es muy alto: más de US\$2,1 billones en compensaciones y US\$ 90 millones en costos directos anuales ${ }^{8}$. Asimismo, la incidencia se ha incrementado, pasando de 141000 casos en 2012 a 184000 en 2013, evidenciando en el 2015 que los DME en conjunto con el estrés y la depresión o la ansiedad representaron la mayoría de los días perdidos debido a problemas de salud relacionados con el trabajo con 9,9 y 9,5 millones de días respectivamente 9 .

Estos trastornos se consideran la nueva epidemia de enfermedades crónicas en el mundo actual ${ }^{10} \mathrm{y}$ su origen es multifactorial ${ }^{8,11}$, por lo que se han abordado de manera interdisciplinar en la búsqueda del máximo confort de las personas en sus estaciones de trabajo ${ }^{12} \mathrm{y}$ el equilibrio entre el sistema hombre, máquina - sistema de trabajo y ambiente ${ }^{13}$. La pérdida de dicho equilibrio puede ocasionar daños en la salud, entre ellos, los DME. Múltiples instrumentos ${ }^{14,15}$ han sido usados para valorar el riesgo biomecánico (carga dinámica ${ }^{16}$ y la carga estática ${ }^{17-19}$ ), entre los que se encuentran el cuestionario Rapid Upper Limb Assesment (RULA) ${ }^{20}$, desarrollado con el objeto de evaluar la exposición de trabajadores a factores de riesgo (biomecánico) que originan una elevada carga postural estática en donde se considera la peor postura adoptada en el lugar de trabajo (la duración, frecuencia y las fuerzas ejercidas cuando se mantienen) que puede ocasionar trastornos en algunos segmentos corporales. A partir de éste, se obtiene una puntuación que determina el nivel de Actuación e indica si la postura es aceptable o no, y si es necesario cambios o rediseños en el puesto de trabajo; este método permite detectar posibles problemas ergonómicos derivados de una carga postural excesiva ${ }^{17}$.

Aunado a lo anterior, la ergonomía participativa surge como una estrategia particularmente atractiva para la prevención de DME a fin de intervenir sobre la exposición a riesgos ergonómicos en el trabajo en los países de renta baja ${ }^{12}$. En dicha estrategia, los diversos actores implicados participan de manera activa en el diagnóstico y en las propuestas para la minimización de factores de riesgo ergonómicos, entre ellos los 
biomecánicos. Algunos métodos han sido usados para conocer la percepción de los síntomas músculoesqueléticos en trabajadores que están expuestos a factores de riesgo de este tipo; ejemplo de ello es el Cuestionario Nórdico ${ }^{21-23}$, el cual identifica los segmentos corporales críticos (en términos de frecuencia y severidad) con existencia de síntomas iniciales, que aún no han constituido enfermedad o no han llevado a consultar al médico. Este instrumento arroja información valiosa para estimar el nivel de riesgos de manera proactiva y permite una actuación precoz (preventiva) ${ }^{24}$; adicional a ello, ha sido usado en múltiples sectores productivos y entre éstos, en operativos $^{25}$, administrativos ${ }^{26}$ y trabajadores de oficina $^{25-28}$. Además, el Cuestionario Nórdico es auto administrado, por lo que puede ser un complemento al método RULA, los cuáles aplicados en conjunto permiten identificar los segmentos corporales críticos en cuanto a nivel de riesgo biomecánico y DME referidos, para así tomar medidas preventivas.

Por lo tanto, se formuló la siguiente pregunta usando la metodología PICO (participantes del interés, la intervención, el control y el resultado primario de interés): ¿Cuál es la información disponible en bases de datos sobre la evaluación de riesgo ergonómico (método RULA) y la percepción de desórdenes musculo esqueléticos (DME) con el Cuestionario Nórdico en trabajadores de diversos sectores económicos, periodo 2014-2015? Para resolver dicha incógnita, se llevó a cabo una revisión sistemática siguiendo la declaración "PRISMA" 29.

\section{MATERIALES Y MÉTODOS}

\section{Estrategia de búsqueda}

Una búsqueda sistemática de la literatura ${ }^{30-32}$ dirigida por las preguntas PICO fue utilizada para identificar artículos sobre el uso del método RULA y Cuestionario Nórdico en trabajadores de diversos sectores productivos. A partir de esta pregunta se realizó una búsqueda de datos en bases de datos como Science direct, Scopus, Pubmed, Springer link, Embase y Ebsco con la combinación de los siguientes términos MeSH de búsqueda: "workers" OR "employment" OR "employed" AND "Nordic/Kuorinka" AND "Rula"; también se realizaron las búsquedas con términos DeCS y términos del lenguaje libre (Tabla 1). La búsqueda se limitó a estudios publicados de enero de 2004 a diciembre de 2015, a fin de recolectar evidencia científica reciente acerca de la valoración del riesgo ergonómico (biomecánico) y percepción de DME. La búsqueda se realizó con la restricción del lenguaje en texto completo en inglés, español o portugués y el alcance fue ampliado según las listas de referencias de los artículos recuperados; los artículos originales fueron cribados manualmente por dos revisores independientes (AEDG) y (DCRR). Si el texto completo de un artículo no estaba disponible en línea para realizar el cribado se procedió a contactar el primer autor del artículo por correo electrónico (e-mail) solicitando una copia de su trabajo.

\section{Selección de estudios}

Los estudios relacionados con la asociación entre el nivel de riesgo ergonómico (carga estática) identificado con RULA y la sintomatología percibida por los trabajadores (Cuestionario Nórdico) se incluyeron si cumplían con las siguientes condiciones: 1) Cualquier estudio que describe la asociación entre nivel de riesgo biomecánico (carga postural estática) y sintomatología osteomuscular con los instrumentos seleccionados para esta revisión; 2) Tipo de publicación: Especializada en ergonomía, salud pública, ciencias médicas, odontología y aplicaciones científicas; 3) Población estudio: Personal que labora en diferentes sectores productivos; 4) Área geográfica: Mundial; 5) Artículos de revistas indexadas de las bases de datos seleccionadas; y 6) Artículos publicados en los cuales simultáneamente se utilizó las dos metodologías (Cuestionario Nórdico y método RULA) sin implementar métodos adicionales. Este último criterio se debe a la limitada concordancia entre estudios sobre asociación del riesgo biomecánico (método RULA) y percepción de DME (Cuestionario Nórdico) en trabajadores de diversos sectores económicos. Por tanto, el objetivo principal de este estudio fue identificar la información disponible en bases de datos sobre evaluación del riesgo biomecánico (RULA) y percepción de DME (Cuestionario Nórdico) en trabajadores de diversos sectores económicos, periodo 2004-2015.

El evento de interés del presente estudio fue revisar la literatura disponible acerca de evaluación del nivel de riesgo biomecánico y la asociación con la percepción de los DME. Particularmente, todos los estudios seleccionados debían contener como principal resultado la asociación antes descrita en cualquier nivel de actuación y reporte de sintomatología. En los estudios que cumplieron con los criterios de selección. La versión de los instrumentos utilizados según se expresa en su metodología fueron: método RULA de McAtamney, et al. ${ }^{33}$ y para el Cuestionario Nórdico de Kuorinka, et al. ${ }^{21}$. 
Salud UIS Vol.49 No.1 Enero - Marzo de 2017

Tabla 1. Registro de revisión de datos.

\begin{tabular}{|c|c|c|c|c|c|c|}
\hline $\begin{array}{l}\text { Nombre de la base de } \\
\text { datos }\end{array}$ & Science direct & Scopus & Pubmed & $\begin{array}{l}\text { Springer } \\
\text { link }\end{array}$ & Embase & Ebsco \\
\hline \multicolumn{7}{|c|}{ 2004-2015 } \\
\hline $\begin{array}{l}\text { Términos de búsqueda } \\
\text { en todas las bases }\end{array}$ & \multicolumn{6}{|c|}{$\begin{array}{l}\text { Término en lenguaje libre } \\
\text { VDT y cuestionario nórdico y RULA; VDT y Kuorinka y RULA; Video terminales y cuestionario } \\
\text { nórdico y RULA; Video terminales y Kuorinka y RULA; Trabajo Video terminales y cuestionario } \\
\text { nórdico y RULA; Trabajo Video terminales y Kuorinka y RULA; Trabajadores de oficina } \\
\text { y cuestionario nórdico y RULA; Trabajadores de oficina y Kuorinka y RULA; Trabajo de } \\
\text { oficina y cuestionario nórdico y RULA; Trabajo de oficina y Kuorinka y RULA; Trabajadores } \\
\text { Administrativos y Cuestionario nórdico y RULA; Trabajadores Administrativos y Kuorinka } \\
\text { y RULA; Trabajo administrativo y Cuestionario nórdico y RULA; Trabajo administrativo y } \\
\text { Kuorinka y RULA; Los trabajadores o empleo o empleado y Cuestionario Nordico y RULA. } \\
\text { Término MeSH } \\
\text { VDT and/or nordic questionnaire and RULA; VDT and/or Kuorinka and RULA; Video terminals } \\
\text { and/or nordic questionnaire and RULA; Video terminals and/or Kuorinka and RULA; Video } \\
\text { terminals job and/or nordic questionnaire and RULA; Video terminals job and/or Kuorinka and } \\
\text { RULA; Office workers and/or nordic Questionnaire and RULA; Office workers and/or Kuorinka } \\
\text { and RULA; Office job and/or nordic Questionnaire and RULA; Office job and/or Kuorinka } \\
\text { and RULA; Administrative workers and/or nordic Questionnaire and RULA; Administrative } \\
\text { workers and/or Kuorinka and RULA; Administrative job and/or nordic Questionnaire and RULA; } \\
\text { Administrative job and/or Kuorinka and RULA; workers OR employment OR employed AND } \\
\text { Nordic AND Rula. } \\
\text { Término DeCS } \\
\text { VDT y/o cuestionario nórdico y RULA; VDT y/o Kuorinka y RULA; Video terminales y/o } \\
\text { cuestionario nórdico y RULA; Video terminales y/o Kuorinka y RULA; Trabajo Video terminales } \\
\text { y/o cuestionario nórdico y RULA; Trabajo Video terminales y/o Kuorinka y RULA; Trabajadores } \\
\text { de oficina y/o cuestionario nórdico y RULA; Trabajadores de oficina y/o Kuorinka y RULA; } \\
\text { Trabajo de oficina y/o cuestionario nórdico y RULA; Trabajo de oficina y/o Kuorinka y RULA; } \\
\text { Trabajadores Administrativos y/o Cuestionario nórdico y RULA; Trabajadores Administrativos } \\
\text { y/o Kuorinka y RULA; Trabajo administrativo y/o Cuestionario nórdico y RULA; Trabajo } \\
\text { administrativo y/o Kuorinka y RULA; Los trabajadores o empleo o empleado y Cuestionario } \\
\text { Nordico y RULA. }\end{array}$} \\
\hline Cualquier campo. & 149 & 22 & 10 & 0 & 443 & 743 \\
\hline Materia (Major Topic) & 45 & 12 & 10 & 0 & 20 & 56 \\
\hline Título & 45 & 12 & 10 & 0 & 20 & 56 \\
\hline Seleccionados & 1 & 6 & 0 & 0 & & 5 \\
\hline
\end{tabular}

Fuente: Elaboración propia.

\section{Extracción de datos}

Se extrajeron los siguientes datos de cada artículo: Autor, año de publicación, país, revista, factor de impacto e indicadores de resultado, sector productivo, el diseño del estudio, afiliación institucional, análisis estadístico utilizado, nivel de riesgo (RULA) y reporte de sintomatología. Los datos fueron registrados en dos bases de datos por dos revisores (AEDG y DCRR) de forma independiente y cualquier discrepancia entre estos dos investigadores (expertos en la materia) se resolvió mediante discusión con un tercer revisor (AIRB), quien también extrajo los datos y participó en la discusión, tomando hizo la decisión final.

\section{Análisis de los datos}

En la fase de análisis de datos se realizó: 1) Análisis bibliométrico con el propósito de brindar un panorama general y un conteo según el lugar de publicación, idioma y tipo de revista; 2) Clasificar el grado de riesgo biomecánico detectado bajo metodología RULA y morbilidad sentida (Cuestionario Nórdico) en trabajadores de diversos sectores económicos; 3 ) Revisar la asociación entre el nivel de riesgo biomecánico (carga postural estática) y la percepción de DME en trabajadores de diversos sectores económicos. Los estudios que cumplieron con los criterios de inclusión y exclusión son en su mayoría de corte trasversal $(n=11$; 
$85 \%)$, seguido de experimentales $(n=1 ; 7.5 \%)$ y de casos y controles $(n=1 ; 7.5 \%)$. Debido a la considerable heterogeneidad y diseño de los estudios encontrados en la búsqueda no fue posible llevar a cabo un metaanálisis para proporcionar un porcentaje global del nivel de riesgo ergonómico como predictor de DME.

\section{RESULTADOS}

En la búsqueda de los estudios elegibles, se introducen las palabras clave mencionadas en Pubmed, Science Direct, Scielo, Redalyc, Springer link, Ebrary, EBSCO,
Proquest, Scopus y se obtuvo un total de 145 estudios. Al aplicar los filtros de idioma y año de publicación, se obtuvieron 144 estudios para ser evaluados, con un total de 38 estudios que cumplieron con los criterios especificados en la sección de metodología tras ser evaluados en texto completo; de estos, 2 artículos fueron excluidos debido a la obtención del texto completo, 10 artículos no utilizaron las dos metodologías en conjunto, 13 documentos no se incluyeron puesto que aplicaron los dos instrumentos en cuestión en combinación con otros métodos (Diagrama 1).

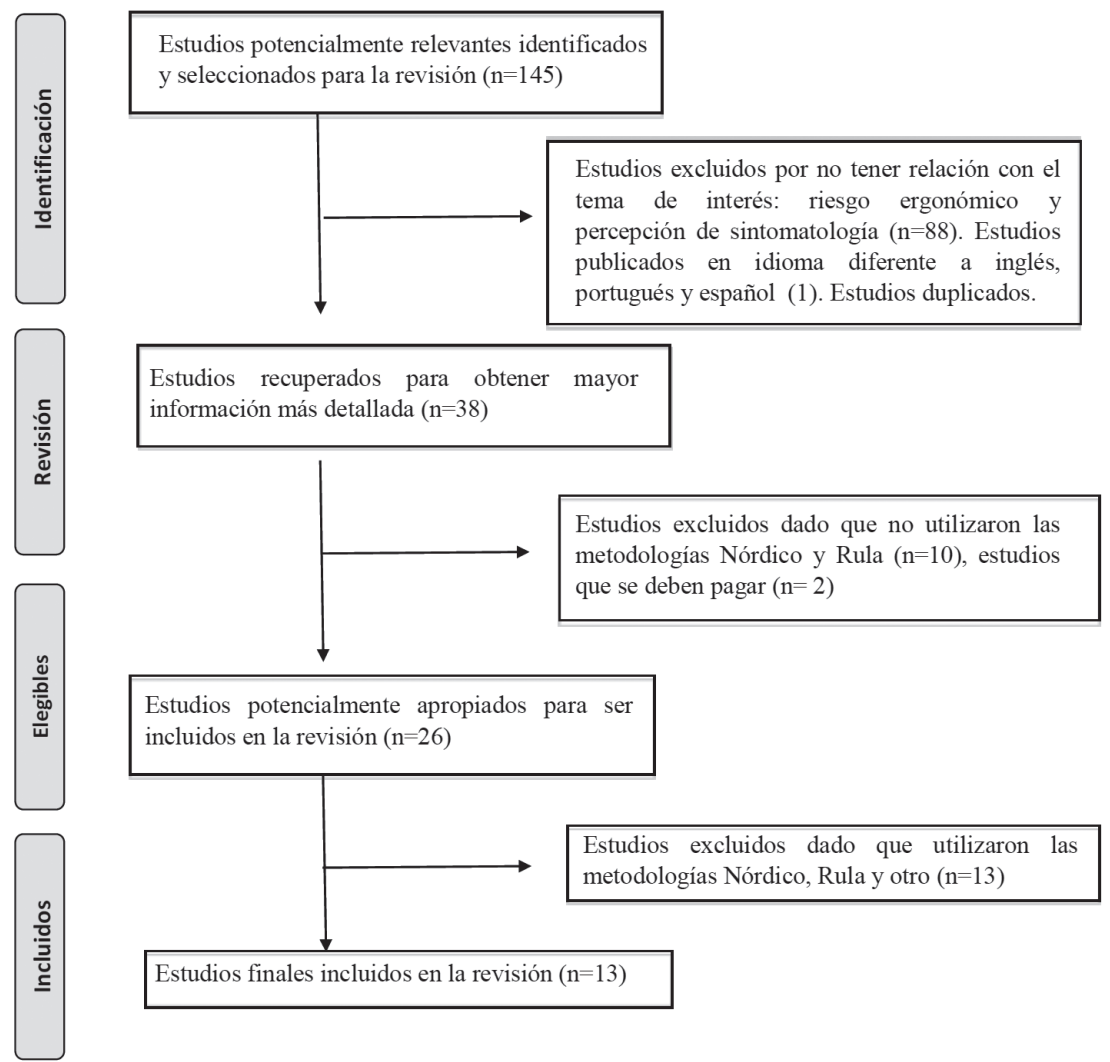

Diagrama 1. Diagrama de flujo del estudio - proceso de selección de los estudios.

Fuente: Elaboración propia.

\section{Análisis bibliométrico}

Se agruparon los resultados en cinco características de acuerdo a representatividad según sectores económicos: 1. Administrativos / video terminales (VDT) $(n=3)$, 2. Operarios de empaque de alimentos $(n=1), 3$. Odontólogos $(n=2), 4$. Operarios de industria $(n=5), 5$. Farmacéutico $(\mathrm{n}=1)$. De los artículos encontrados, el $38,46 \%$ corresponden al año 2015 y $7.69 \%(n=1)$ para los años 2004, 2005, 2006, 2007, 2008, 2010, 2013 у 2014 (Tabla 2). El rango de autores por publicación es de dos a seis, con un promedio de 3.6 autores por publicación; se identificaron 30 autores de género masculino y 12 de femenino, lo que implica que aproximadamente por cada 1 mujeres hay 3 hombres que publicaron sobre la temática estudiada.

Según el lugar de publicación se identificaron 22 autores en Irán, 6 en Malasia, 8 en India y en menor número en Israel (2), México (2) y Colombia (2), quienes estaban adscritos a 32 instituciones y universidades lo que refleja el trabajo interinstitucional (Tabla 2). Dos trabajos cuentan con colaboración internacional: Amin, et al. (2009) establecida entre Malasia y Canadá, y 
Dianat, et al. (2015) realizado en colaboración de Irán y Reino Unido. Todos los artículos cuentan con coautoría. En lo relacionado al idioma de publicación, la mayor parte de los artículos están publicados en idioma inglés $(n=11,84.6 \%)$ y el resto en español $(n=15,4 \%) .12$ artículos fueron publicados en su idioma oficial, 10 en inglés (India 2; Irán 6 y Malasia 2), 2 en español (México 1 y Colombia 1) y 1 artículo en inglés, siendo el idioma oficial persa. No se encontraron estudios en portugués (completos) Figura 1.

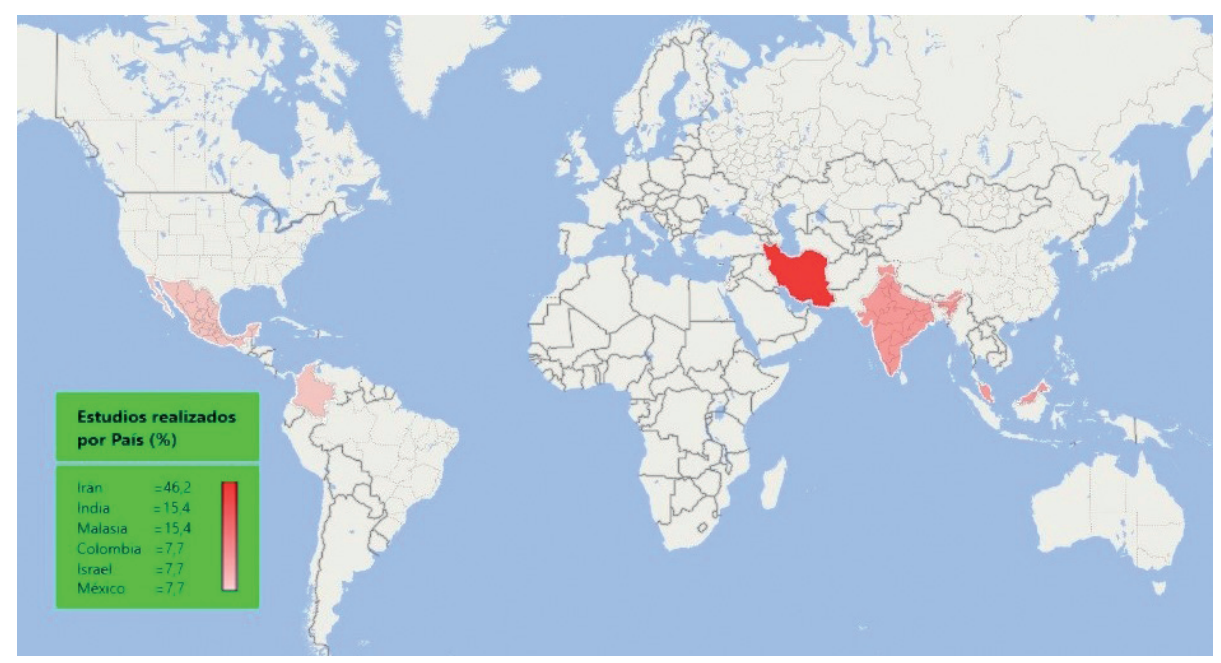

Figura 1. Número de estudios revisados por país.

Fuente: Elaboración propia.

\section{Factor de impacto}

La publicación de los artículos por tipo de revista es diversa. En medicina se identificaron cinco publicaciones, dos en salud pública, tres en ergonomía, una de aplicaciones científicas, una de investigación y una en odontología; según la clasificación del Factor de Impacto Scimago Journal \& Country Rank, se identificaron cuatro revistas en Q2(Journal of Mechanical Engineering and Sciences, Journal of Environmental and Public Health, International Journal of Industrial Ergonomics y Indian Journal of Community Medicine), tres en Q3 de psicología (International Journal of Occupational Safety and Ergonomics (JOSE), Indian Journal of Dental Research: Official Publication of the Indian Society for Dental Research, Pakistan Journal of Medical Sciences y Journal of Applied Science), 2 en Q4 de medicina (Malaysian Journal of Medicine and Health Sciences y Indian Journal of Occupational and Environmental Medicine) y una en Q1 en Ergonomía (Applied Ergonomics). Según Publindex la revista Investigaciones Andina Clasificó en A2 y es una revista no cuenta con factor de impacto.

\section{Indicadores de desempeño}

Se identificó que el trabajo de Shuvala, et al. (2005) tiene 60 citas identificadas en ISSI, seguidos por Choobineha, et al. (2004) con 53 citas, Choobineh, Tabatabaei \& Cols (2007) con 41 citas; Pourmahabadian, et al.
(2008) con 12 citas; Pourmahabadian, et al. (2006) con 10 citas; Arenas, et al. (2013) con 8 citas; así como Dianat, et al. (2015), Golchha, et al. (2015) con cinco citas respectivamente; Rafie, et al. (2015) con dos citas; Anita, et al. (2014) con una cita. El resto de autores no son reconocidos en la base de ISSI.

\section{Método y temas centrales de los estudios incluidos en la revisión}

Los 13 estudios incluidos en la revisión variaron en términos de temas abordados, mientras los métodos y técnicas utilizadas se mantuvieron constantes; sin embargo, todos usaron una metodología cuantitativa. Las técnicas utilizadas en los estudios cuantitativos, diseño, muestra, población y análisis de resultados se describen en la Tabla 2. Tres estudios no describen el análisis estadístico y uno la selección de la muestra.

\section{Análisis de resultados}

Resultados trabajadores administrativos: Tras analizar los estudios, se encontró que para la metodología RULA en los trabajadores administrativos, la puntuación Global se clasificó en Nivel 4; por lo cual, en las estaciones de trabajo evaluadas se hizo necesaria profundizar en el estudio, así como realizar las adaptaciones según fuera el caso. De otro lado, al depurar la información por la sintomatología, se encontraron prevalencias altas según Shuval, et al. ${ }^{34} \mathrm{y}$ 
Rodríguez, et al. ${ }^{27}$ en cuello 46,9\% y 47,6\%, seguido por Hombro $27,1 \%$ y $47,6 \%$, espalda baja $46,9 \%$, espalda alta $42.7 \%$ y mano muñeca $32,1 \%$ Tabla 3 .

Resultados trabajadores operativos: En lo referente a estudios que incluían trabajadores operativos se encontró en la puntuación de nivel de acción 4 (23\%) arrojando cambios urgentes en la tarea. Con relación a la prevalencia de síntomas según Nawawi et al. ${ }^{35}$, Golchha, et al. ${ }^{36}$ y Ghosh et al. ${ }^{37}$ se encontró en cuello desde $48,3 \%$ hasta $92,9 \%$; espalda alta 7,7\%-48,4\% y espalda baja $65,9 \%-75 \%$; hombro $10,6 \%-79,5 \%$; mano muñeca $32,1 \%-45 \%$; y piernas $59,3 \%$ (Tabla 3).

No obstante, un $46 \%$ de los estudios arrojaron nivel de actuación 3 para RULA, requiriéndose el rediseño de la tarea. Con relación a la prevalencia de síntomas, según Abd Rahman et al. (2014) $)^{38}$ (38), Dianat, et al. ${ }^{39}$, Choobineh et al. $(2014)^{40}$, Arenas-Ortiz \& CantúGómez $(2015)^{41}$, Choobineh, et al. ${ }^{42}$ y Shuval, et al. ${ }^{34}$, se encontró en cuello desde $32,2 \%$ hasta $64,7 \%$; espalda alta 31\%-66.7\%; espalda baja $37.8 \%-66.7 \%$; hombro 27.8\%-73\%; mano muñeca $32,1 \%-65.5 \%$ (Tabla 3).

Con relación al nivel de actuación 2 para RULA (pueden requerirse cambios en la tarea, es conveniente profundizar en el estudio) y teniendo en cuenta la prevalencia de síntomas según Pourmahabadian, et al. ${ }^{43}$, se encontró en espalda baja $54.8 \%$, miembros inferiores o muslos $26.2 \%$ y espalda alta un $19 \%$, en este último segmento en trabajadores de embalaje (Tabla 3 ).

Resultados estudios trabajadores administrativostrabajadores operativos: En el estudio de casos (operativos) y control (administrativos), el 52\% puntuaron en nivel 3 para RULA. Con relación a la prevalencia de síntomas según Pourmahabadian, et al., se encontró en el personal operativo de prensa mayor prevalencia de síntomas en espalda $(60 \%)$, rodillas (40\%), cuello $(32 \%)$ y hombros $(22 \%)$ frente a los trabajadores administrativos, en los cuales los segmentos con mayores prevalencias fueron: cuello (44\%), rodillas (24\%) y piernas (22\%) (Tabla 3).

Tabla 2. Metodología utilizada en estudios seleccionados, 2004-2015.

\begin{tabular}{|c|c|c|c|c|c|c|}
\hline AUTOR & $\begin{array}{l}\text { TIPO DE } \\
\text { ESTUDIO }\end{array}$ & INSTRUMENTO & $\begin{array}{l}\text { POBLACIÓN } \\
\text { FUENTE Y PAÍS }\end{array}$ & MUESTRA & MUESTREO & ANÁLISIS ESTADÍSTICO \\
\hline Anita et al (2014) & $\begin{array}{c}\text { Corte } \\
\text { trasversal }\end{array}$ & $\begin{array}{c}\text { Método Rula } \\
\text { y Cuestionario } \\
\text { Nórdico }\end{array}$ & $\begin{array}{l}\text { MALASIA, } \\
\text { trabajadores } \\
\text { en una línea } \\
\text { de ensamblaje } \\
\text { de la industria } \\
\text { automotriz }\end{array}$ & 232 & MAS & $\begin{array}{l}\text { Estadística descriptiva para edad, género, estado civil, } \\
\text { dominancia manual, nivel educativo, tipo de trabajo, } \\
\text { estilos de vida, sintomatología y RULA; Chi cuadrado } \\
\text { para edad, género, estado civil, dominancia manual, } \\
\text { nivel educativo, tipo de trabajo vs sintomatología, } \\
\text { RULA vs sintomatología; análisis de regresión } \\
\text { logística edad, tipo de trabajo, nivel de riesgo RULA } \\
\text { vs sintomatología. }\end{array}$ \\
\hline $\begin{array}{l}\text { Dianat et al } \\
\text { (2015) }\end{array}$ & $\begin{array}{c}\text { Estudio } \\
\text { transversal, } \\
\text { descriptivo- } \\
\text { estudio } \\
\text { analítico }\end{array}$ & $\begin{array}{c}\text { Método Rula } \\
\text { y Cuestionario } \\
\text { Nórdico }\end{array}$ & $\begin{array}{l}5000 \text { operarios, } \\
\text { en } 3 \text { provincias } \\
\text { del oeste de Irán } \\
\text { (p. ej. Azerbaiyán } \\
\text { Oriental, } \\
\text { Kermanshah y } \\
\text { Kurdistán) }\end{array}$ & $\begin{array}{c}251 \text { operadores de } \\
\text { máquinas de coser } \\
\text { iraní. ( } 151 \text { hombres } \\
\text { y } 100 \text { mujeres) }\end{array}$ & MMAS & $\begin{array}{l}\text { Estadística descriptiva para género, edad, IMC, estado } \\
\text { civil, nivel educativo, actividad física, consumo de } \\
\text { cigarrillos, años de experiencia, horas de trabajo diario, } \\
\text { horas de trabajo semanal, rapidez en el trabajo, nivel } \\
\text { de satisfacción en el trabajo, uso de sillas ajustables, } \\
\text { satisfacción entre las tareas que debe desarrollar, } \\
\text { RULA); análisis de regresión logística múltiple } \\
\text { de factores (género, edad, IMC, estado civil, nivel } \\
\text { educativo, actividad física, consumo de cigarrillos, años } \\
\text { de experiencia, horas de trabajo diario, horas de trabajo } \\
\text { semanal, rapidez en el trabajo, nivel de satisfacción en } \\
\text { el trabajo, uso de sillas ajustables, satisfacción entre las } \\
\text { tareas que debe desarrollar, RULA ) asociados con la } \\
\text { sintomatología de cuello, hombro y mano/muñeca. }\end{array}$ \\
\hline $\begin{array}{l}\text { Nawawi, et al } \\
(2015)\end{array}$ & $\begin{array}{c}\text { Corte } \\
\text { trasversal }\end{array}$ & $\begin{array}{c}\text { Método Rula } \\
\text { y Cuestionario } \\
\text { Nórdico. }\end{array}$ & $\begin{array}{l}\text { Operadores de } \\
\text { máquinas de coser } \\
\text { de Costa Este, } \\
\text { Costa oeste, así } \\
\text { como el Norte y el } \\
\text { sur de la península } \\
\text { de Malasia }\end{array}$ & $\begin{array}{c}337 \text { operadores } \\
\text { de máquinas de } \\
\text { coser hombres } \\
\mathrm{n}=122, \text { mujeres }= \\
215 \text {, edades entre } \\
18-54 \text { años de } \\
\text { edad; la media de } \\
\begin{array}{c}30.74 \pm 8.44) \text { salario } \\
(\mathrm{n}=246) \text { y destajo } \\
(\mathrm{n}=91) .\end{array} \\
\end{array}$ & MAE. & $\begin{array}{l}\text { Estadística descriptiva para tiempo de trabajo diario } \\
\text { y semanal, años de experiencia laboral, descansos, } \\
\text { sensación de agotamiento y presión, experiencia de la } \\
\text { exposición física y forma de pagos de salarios; Chi- } \\
\text { cuadrado para evaluar las asociaciones entre variables } \\
\text { como el tipo de salario y factores de riesgo ergonómico; } \\
\text { Física (tipos de salarios y factores de riesgo físico) y } \\
\text { tipos de salarios y síntomas musculo esqueléticos); } \\
\text { modelo de regresión logística entre tipos de salario y } \\
\text { prevalencia de síntomas. }\end{array}$ \\
\hline $\begin{array}{l}\text { Choobineha et al } \\
\text { (2004) }\end{array}$ & $\begin{array}{l}\text { Estudio de } \\
\text { Intervención }\end{array}$ & $\begin{array}{l}\text { Método Rula } \\
\text { y Cuestionario } \\
\text { Nórdico }\end{array}$ & Irán & $\begin{array}{l}72 \text { operarios } \\
\text { de zurcido } \\
\text { de alfombras } \\
\text { distribuidos en } \\
12 \text { estaciones de } \\
\text { trabajo }\end{array}$ & NE. & $\begin{array}{l}\text { Estadísticos descriptivos para edad, años de } \\
\text { experiencia, horas de trabajo semanal, sintomatología } \\
\text { y RULA); Prueba de proporciones de una muestra } \\
\text { entre las condiciones de trabajo (forma tradicional y } \\
\text { una mesa) vs diferentes tipos de trabajos (hechura de } \\
\text { alfombra, unión de alfombra, embalaje, anudado y } \\
\text { tejido). }\end{array}$ \\
\hline
\end{tabular}


Salud UIS Vol.49 No.1 Enero - Marzo de 2017

\begin{tabular}{|c|c|c|c|c|c|c|}
\hline AUTOR & $\begin{array}{c}\text { TIPO DE } \\
\text { ESTUDIO }\end{array}$ & INSTRUMENTO & $\begin{array}{c}\text { POBLACIÓN } \\
\text { FUENTE Y PAÍS }\end{array}$ & MUESTRA & MUESTREO & ANÁLISIS ESTADÍSTICO \\
\hline $\begin{array}{l}\text { Golchha et al } \\
\text { (2015). }\end{array}$ & $\begin{array}{l}\text { Corte } \\
\text { trasversal }\end{array}$ & $\begin{array}{l}\text { Método Rula } \\
\text { y Cuestionario } \\
\text { Nórdico. }\end{array}$ & $\begin{array}{c}\text { Odontólogos de } \\
\text { Nueva Delhi, India }\end{array}$ & $\begin{array}{l}104 \text { odontólogos, } \\
\text { dentro de ellos } \\
70 \text { hombres y } 34 \\
\text { mujeres de Nueva } \\
\text { Delhi }\end{array}$ & MAS & $\begin{array}{l}\text { Descriptivo para género, Rula y sintomatología; } \\
\text { Chi Cuadrado de Pearson para género, RULA vs } \\
\text { sintomatología. }\end{array}$ \\
\hline
\end{tabular}

Rodríguez \& Dimaté (2015)

$\begin{array}{cc}\text { Corte } & \text { Método Rula } \\ \text { trasversal } & \text { y Cuestionario } \\ \text { Nórdico. }\end{array}$

Nórdico.
Administrativos de 96 trabajadores una Universidad administrativos de Bogotá, Colombia la Universidad,

Estadísticos Descriptivos para edad, peso, estatura, IMC, estado civil, ocupación, escolaridad, antecedentes familiares, pausas activas programadas, C. dominancia manual, sintomatología y RULA; Medidas de Riesgo (OR) (Woolf y Cornfield) entre edad, peso, estatura, IMC, estado civil, ocupación, escolaridad, antecedentes familiares, pausas activas programadas, dominancia manual, RULA vs sintomatología.

\begin{tabular}{|c|c|c|c|c|c|c|}
\hline $\begin{array}{l}\text { Pourmahabadian } \\
\text { \& Azam (2006) }\end{array}$ & $\begin{array}{l}\text { Casos y } \\
\text { control }\end{array}$ & $\begin{array}{l}\text { Método Rula } \\
\text { y Cuestionario } \\
\text { Nórdico. }\end{array}$ & $\begin{array}{c}\text { Industria de } \\
\text { fabricación de } \\
\text { automóviles en } \\
\text { Teherán durante } \\
2004-2005 . \\
50 \text { trabajadores } \\
\text { de prensa y } 50 \\
\text { administrativos. }\end{array}$ & $\begin{array}{c}\text { Cincuenta } \\
\text { trabajadores } \\
\text { hombres de prensa } \\
\text { (en flexión, impacto } \\
\text { y tiendas prensa } \\
\text { hidráulica) se } \\
\text { inscribieron en } \\
\text { el estudio como } \\
\text { grupo de casos } \\
\text { y } 50 \text { personal } \\
\text { administrativo } \\
\text { como grupo control } \\
\text { en una industria } \\
\text { de fabricación de } \\
\text { automóviles en } \\
\text { Teherán durante } \\
\text { 2004-2005 }\end{array}$ & C. & $\begin{array}{l}\text { Estadística descriptiva para prevalencia de síntomas a } \\
12 \text { meses, RULA, tipo de trabajo; Pruebas de Fisher } \\
\text { para sintomatología y RULA (Brazo, muñeca y grupo } \\
\text { B); Chi Cuadrado para sintomatología y RULA (cuello } \\
\text { y tronco). }\end{array}$ \\
\hline $\begin{array}{l}\text { Arenas-Ortiz \& } \\
\text { Cantú-Gómez } \\
(2013)\end{array}$ & $\begin{array}{c}\text { Corte } \\
\text { trasversal }\end{array}$ & $\begin{array}{l}\text { Método Rula } \\
\text { y Cuestionario } \\
\text { Nórdico. }\end{array}$ & $\begin{array}{l}\text { México, Zapopan, } \\
\text { Jalisco }\end{array}$ & $\begin{array}{c}90 \text { los trabajadores } \\
\text { de una planta } \\
\text { de producción } \\
\text { donde se elabora } \\
\text { y empaqueta frijol } \\
\text { para consumo } \\
\text { instantáneo en } \\
\text { Zapopan, Jalisco } \\
\end{array}$ & C. & $\begin{array}{l}\text { Estadística descriptiva para género, tipo de trabajo, } \\
\text { RULA y sintomatología. }\end{array}$ \\
\hline $\begin{array}{l}\text { Pourmahaba et al } \\
\text { (2008). }\end{array}$ & $\begin{array}{l}\text { Corte } \\
\text { trasversal }\end{array}$ & $\begin{array}{l}\text { Método Rula } \\
\text { y Cuestionario } \\
\text { Nórdico. }\end{array}$ & $\begin{array}{l}\text { Trabajadores de } \\
\text { tres unidades } \\
\text { farmacéuticas, } \\
\text { productos } \\
\text { de higiene y } \\
\text { administrativos. }\end{array}$ & $\begin{array}{c}950 \text { trabajadores, } \\
66,7 \text { hombres } \\
\text { y } 33,3 \text { mujeres, } \\
\text { en tres unidades } \\
\text { farmacéuticas, } \\
\text { productos } \\
\text { de higiene y } \\
\text { administrativos. } \\
\text { En este estudio, } \\
84 \text { trabajadores } \\
\text { masculinos y } \\
\text { femeninos de } \\
\text { las operaciones } \\
\text { de embalaje } \\
\text { (antibióticos, Tablet, } \\
\text { ampolla, jarabe y } \\
\text { povidona) fueron } \\
\text { seleccionados } \\
\text { aleatoriamente y } \\
\text { participado. }\end{array}$ & MAS & $\begin{array}{l}\text { Estadística descriptiva para género, tipo de trabajo, } \\
\text { sintomatología y RULA; Chi Cuadrado de Pearson } \\
\text { (1df) para género, puntuación RULA, tipo de trabajo } \\
\text { vs sintomatología; prueba exacta de Fischer para } \\
\text { género, puntuación RULA vs sintomatología; Pruebas } \\
\text { de hipótesis }\left(\mathrm{P}^{2}\right) \text { puntuación RULA vs sintomatología. }\end{array}$ \\
\hline $\begin{array}{l}\text { Choobineh et al } \\
\text { (2007). }\end{array}$ & $\begin{array}{l}\text { Corte } \\
\text { trasversal }\end{array}$ & $\begin{array}{l}\text { Método Rula } \\
\text { y Cuestionario } \\
\text { Nórdico. }\end{array}$ & $\begin{array}{l}\text { Trabajadores de } \\
\text { una empresa de } \\
\text { comunicaciones en } \\
\text { IRAN }\end{array}$ & $\begin{array}{l}85 \text { trabajadores } \\
\text { de la Línea de } \\
\text { producción } \\
\text { del circuito de } \\
\text { televisión } \\
\end{array}$ & MAS & $\begin{array}{l}\text { Estadística descriptiva para edad, permanecía en el } \\
\text { empleo, sintomatología y RULA; Test de Mann- } \\
\text { Whitney para permanencia en el empleo, género, Nivel } \\
\text { de riesgo RULA vs sintomatología. }\end{array}$ \\
\hline $\begin{array}{l}\text { Rafie et al } \\
(2015) \text {. }\end{array}$ & $\begin{array}{l}\text { Corte } \\
\text { trasversal }\end{array}$ & $\begin{array}{l}\text { Método Rula } \\
\text { y Cuestionario } \\
\text { Nórdico. }\end{array}$ & Kerman & $\begin{array}{l}130,84 \text { hombres y } \\
46 \text { mujeres. }\end{array}$ & C. & $\begin{array}{l}\text { Estadística descriptiva para sexo, IMC, edad, } \\
\text { experiencia en años, horas de trabajo por semana, } \\
\text { número de pacientes por semana, sintomatología y } \\
\text { RULA; Chi Cuadrado para RULA vs tipos de trabajo y } \\
\text { género, características de la ocupación vs síntomas; T } \\
\text { Student para género vs RULA. }\end{array}$ \\
\hline
\end{tabular}


Percepción de desórdenes musculoesqueléticos y aplicación del método RULA en diferentes sectores productivos: una revisión sistemática de la literatura

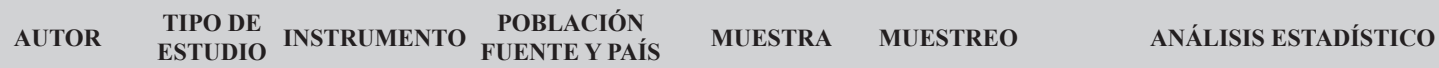

$\begin{array}{lcccc}\text { Shuvala \& } & \text { Corte } & \text { Método Rula } & \text { y trabajadores de } & \\ \text { Donchinb (2005). } & \text { trasversal } & \text { y Cuestionario } & 84 \text { trabajodores de } \\ \text { Nórdico. } & \text { VDT; Israel } & 91 \text { trabajado } & \text { VDT }\end{array}$

Estadística Descriptiva para edad, genero, estado civil, número de hijos, uso de gafas, uso de Computador los fines de semana, Actividad Física, Modificación de Puesto de Trabajo, Características Ocupacionales (Descripción de Trabajo, Tiempo de trabajo diario,

C. Tiempo de Trabajo Semanal, Tiempo de experiencia en la compañía, experiencia en $\mathrm{Hi}-\mathrm{Tech}$, tareas adicionales), sintomatología y RULA; Regresión logística y ANOVA para las variables anteriores;

\begin{tabular}{|c|c|c|c|c|c|c|}
\hline $\begin{array}{l}\text { Ghosh et al } \\
(2010) \text {. }\end{array}$ & $\begin{array}{c}\text { Corte } \\
\text { trasversal }\end{array}$ & $\begin{array}{c}\text { Método Rula } \\
\text { y Cuestionario } \\
\text { Nórdico. }\end{array}$ & $\begin{array}{c}\text { Davangere distrito } \\
\text { de Karnataka. }\end{array}$ & $\begin{array}{l}120 \text { hombres } \\
\text { orfebres informales. }\end{array}$ & MAS & $\begin{array}{l}\text { Análisis Descriptivo para sintomatología y Método } \\
\text { RULA. }\end{array}$ \\
\hline
\end{tabular}

C: conveniencia. NE: no Especifico. MAE: Muestreo Aleatoria Estratificado. MAS: Muestreo aleatorio simple. MMAS=multinivel muestreo aleatorio simple.

Fuente: Elaboración propia.

Tabla 3. Estudios sobre la percepción de DME y método rula medidas tomadas.

\begin{tabular}{|c|c|c|c|c|c|c|c|c|c|c|c|c|c|}
\hline \# & AUTOR & $\begin{array}{c}\text { NIVEL DE RIESGO } \\
\text { RULA }\end{array}$ & $\begin{array}{c}\text { REPORTE DE } \\
\text { SINTOMATOLOGÍA } \\
\text { KUORINKA }\end{array}$ & Variable & Segmento & $\chi^{2}$ & $\begin{array}{l}\text { Fisher } \\
\chi^{2} \text { Test } \\
\text { Exact }\end{array}$ & $\mathbf{P}$ & $\mathbf{P 2}$ & $\begin{array}{c}\text { P } \\
\text { E-Value }\end{array}$ & OR & $\begin{array}{l}\text { IC - } \\
95 \%\end{array}$ & $\begin{array}{l}\text { Nivel de } \\
\text { evidencia } \\
\text { Sackett y } \\
\text { OCEBM }\end{array}$ \\
\hline \multirow{5}{*}{1} & \multirow{5}{*}{ 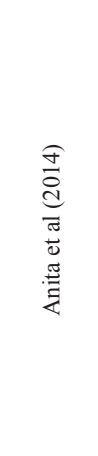 } & \multirow{5}{*}{$\begin{array}{c}\text { La Metodología } \\
\text { RULA fue aplicada } \\
\text { para la evaluar la } \\
\text { peor postura, se } \\
\text { encontró en los } \\
\text { niveles de riesgo: } \\
\text { Bajo e Intermedio + } \\
\text { síntomas } 26(42,6 \%) \\
\text { - síntomas } 35(57,3 \%) \\
\text { Alto + síntomas } 87 \\
(87,0 \%) \text { - síntomas } \\
13(13,0 \%) \text { muy alta } \\
\text { + síntomas } 69(97,2 \%) \\
\text { - síntomas } 2(2.8 \%)\end{array}$} & \multirow{5}{*}{$\begin{array}{c}\text { La prevalencia de MSD } \\
78,2 \% \text {, espalda baja } \\
118(50,9 \%) \text {, seguido } \\
\text { por el hombro } 88 \\
(37,9 \%), \text { la muñeca } \\
\text { / mano } 79(34,1 \%), \\
\text { el cuello } 77(32,2 \%), \\
\text { espalda alta } 72(31 \%) \text {, } \\
\text { la rodilla } 59(25,4 \%), \\
\text { el tobillo / pies } 56 \\
(24,1 \%), \text { cadera / } \\
\text { muslo } 38(16,4 \%) \text { y el } \\
\text { codo } 21(9,1 \%) .\end{array}$} & $>25$ años & $\begin{array}{l}\text { Probabilidad } \\
\text { DME }\end{array}$ & - & - & - & - & - & 2,25 & $\begin{array}{l}1,14- \\
4,46\end{array}$ & \multirow{5}{*}{$\mathrm{C} 4$} \\
\hline & & & & $\begin{array}{l}\text { Antigüedad > } \\
3 \text { años }\end{array}$ & $\begin{array}{l}\text { Probabilidad } \\
\text { DME }\end{array}$ & - & - & - & - & - & 2,44 & $\begin{array}{l}1,04- \\
5,63\end{array}$ & \\
\hline & & & & $\begin{array}{c}\text { RULA } 4 \\
\text { y3 (riesgo } \\
\text { muy alto e } \\
\text { intermedio) }\end{array}$ & $\begin{array}{l}\text { Probabilidad } \\
\text { DME }\end{array}$ & - & - & - & - & - & 69,38 & $\begin{array}{l}14,51- \\
331,73\end{array}$ & \\
\hline & & & & $\begin{array}{c}\text { RULA } 4 \text { y }<3 \\
\text { (riesgo muy } \\
\text { alto y bajo). }\end{array}$ & $\begin{array}{l}\text { Probabilidad } \\
\text { DME }\end{array}$ & - & - & - & - & - & 12.42 & $\begin{array}{l}5,21- \\
29,58\end{array}$ & \\
\hline & & & & RULA & Sintomatología & & $\begin{array}{l}65,37 \\
\text { d.f. }=2\end{array}$ & - & - & 0,001 & - & - & \\
\hline \multirow{12}{*}{2} & \multirow{12}{*}{ 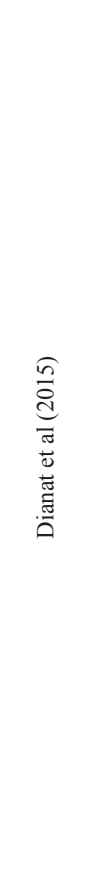 } & \multirow{12}{*}{$\begin{array}{l}\text { Se encontraron } \\
\text { significancias } \\
\text { estadísticas al hacer } \\
\text { el cruce entre el } \\
\text { nivel de riesgo } \\
\text { RULA, variables } \\
\text { sociodemográficas } \\
\text { como género, edad, } \\
\text { antigüedad, etc.; así } \\
\text { mismo al hacer el } \\
\text { cruce entre RULA y } \\
\text { sintomatología. }\end{array}$} & $\begin{array}{c}\text { Al hacer los } \\
\text { descriptivos se }\end{array}$ & & Mano Muñeca U & - & - & 0.004 & - & - & 0.63 & $\begin{array}{l}0.38- \\
0.95\end{array}$ & \multirow{12}{*}{$\mathrm{C} 3$} \\
\hline & & & $\begin{array}{l}\text { encontró que: Espalda } \\
\text { Alta } n=148(58.9 \%) \text {, } \\
\text { Cuello } n=136(54.1 \%) \text {, }\end{array}$ & $\begin{array}{l}\text { Género } \\
\text { Hombre }\end{array}$ & Espalda Alta U & - & - & 0.001 & - & - & 11.4 & $\begin{array}{l}5.75- \\
20.5\end{array}$ & \\
\hline & & & $\begin{array}{l}\text { Mano muñeca } n=101 \\
(40.2 \%), \text { la espalda }\end{array}$ & & Espalda Alta M & - & - & 0.001 & - & - & 10.9 & $\begin{array}{l}5.1- \\
18.2\end{array}$ & \\
\hline & & & $\begin{array}{l}\text { baja }(37,8 \%) \text { y los } \\
\text { hombros }(27,8 \%) \text {. }\end{array}$ & & Cuello U & - & - & 0.044 & - & - & 1.03 & $\begin{array}{l}1.01- \\
1.06\end{array}$ & \\
\hline & & & $\begin{array}{c}\text { prevalencia en todas } \\
\text { edades ( }>18 \text { años) }\end{array}$ & Edad & Hombro U & - & - & 0.001 & - & - & 1.04 & $\begin{array}{l}1.01- \\
1.07\end{array}$ & \\
\hline & & & $\begin{array}{c}(\mathrm{n}=70) 27,8 \% \text { Dolor } \\
\text { mano muñeca Vs } \\
\text { prevalencia en todas }\end{array}$ & IMC & Espalda Alta U & - & - & 0.001 & - & - & 0.88 & $\begin{array}{l}0.82- \\
0.94\end{array}$ & \\
\hline & & & $\begin{array}{c}\text { edades ( }>18 \text { años) } \\
(\mathrm{n}=101) 27,8 \% \text { Dolor }\end{array}$ & $\begin{array}{l}\text { Estado Civil } \\
\text { Casado }\end{array}$ & Cuello U & - & - & 0.035 & - & - & 1.93 & $\begin{array}{l}1.04- \\
3.58\end{array}$ & \\
\hline & & & $\begin{array}{l}\text { rodillas } v \text { s prevalencia } \\
\text { en todas edades }(>18 \\
\text { años) }(n=51) 20.3 \% \text {; }\end{array}$ & $\begin{array}{c}\text { Nivel } \\
\text { Educativo }\end{array}$ & Hombro U & - & - & 0.002 & - & - & 0.24 & $\begin{array}{c}0.09- \\
0.58\end{array}$ & \\
\hline & & & $\begin{array}{c}\text { Síntomas en cuello y } \\
\text { severidad calificado de } \\
0-53.3(0.93) \text {. }\end{array}$ & Secundaria & Espalda Alta U & - & - & 0.001 & - & - & 0.08 & $\begin{array}{c}0.03- \\
0.20\end{array}$ & \\
\hline & & & $\begin{array}{c}\text { Síntomas en espalda } \\
\text { baja y severidad }\end{array}$ & $\begin{array}{c}\text { Deporte } \\
\text { Regular Act. }\end{array}$ & Mano Muñeca U & - & - & 0.001 & - & - & 0.29 & $\begin{array}{c}0.15- \\
0.55\end{array}$ & \\
\hline & & & $\begin{array}{c}\text { calificado de } 0-53.8 \\
(0.92)\end{array}$ & Física & Mano Muñeca M & - & - & 0.001 & - & - & 0.3 & $\begin{array}{c}0.15- \\
0.60\end{array}$ & \\
\hline & & & $\begin{array}{c}\text { Síntomas en rodillas y } \\
\text { severidad calificado de } \\
0-52.6(0.89) .\end{array}$ & Cigarrillo & Espalda Alta U & - & - & 0.001 & - & - & 6.06 & $\begin{array}{l}2.93- \\
12.5\end{array}$ & \\
\hline
\end{tabular}


Salud UIS Vol.49 No.1 Enero - Marzo de 2017

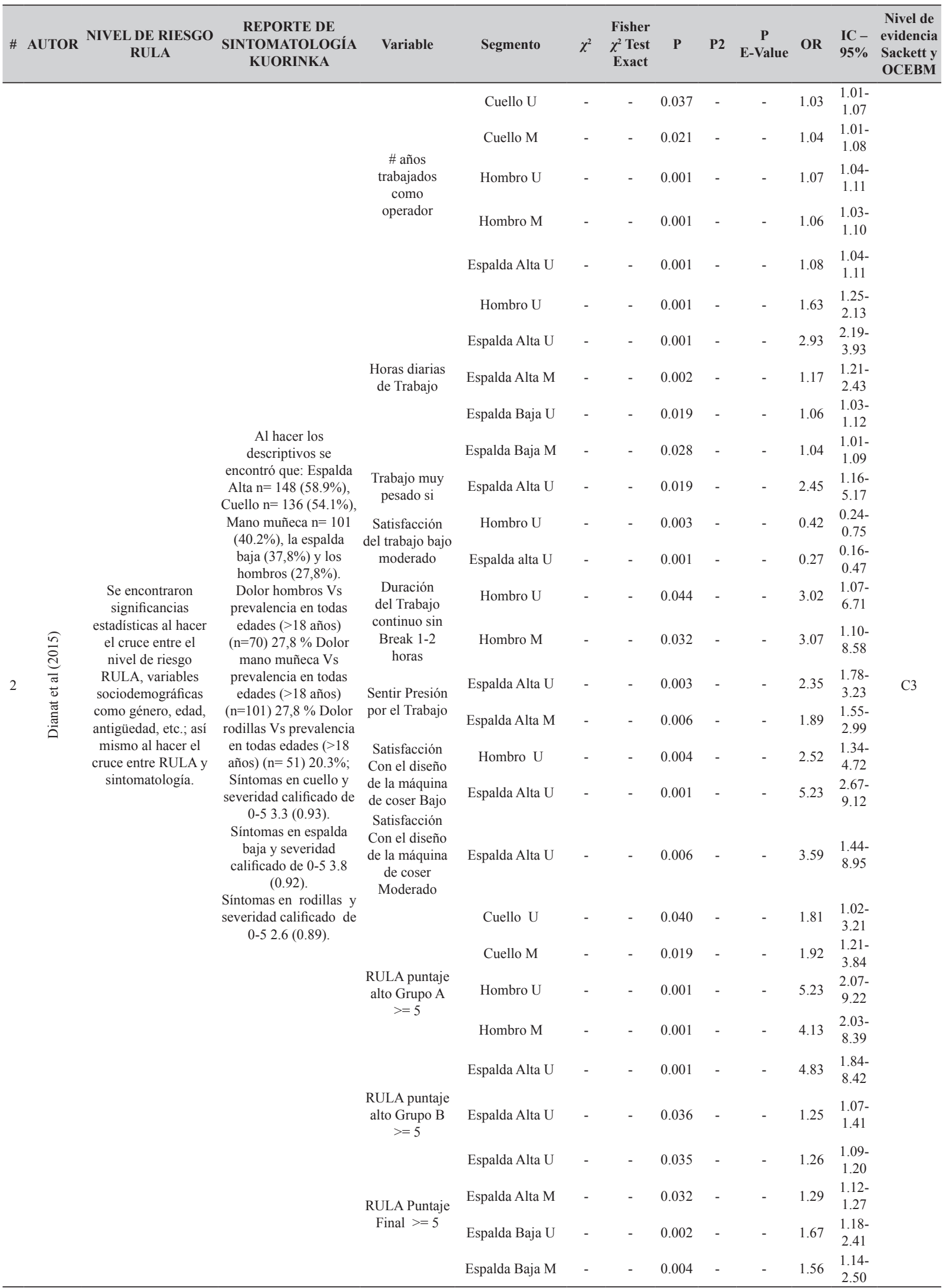


Percepción de desórdenes musculoesqueléticos y aplicación del método RULA en diferentes sectores productivos: una revisión sistemática de la literatura

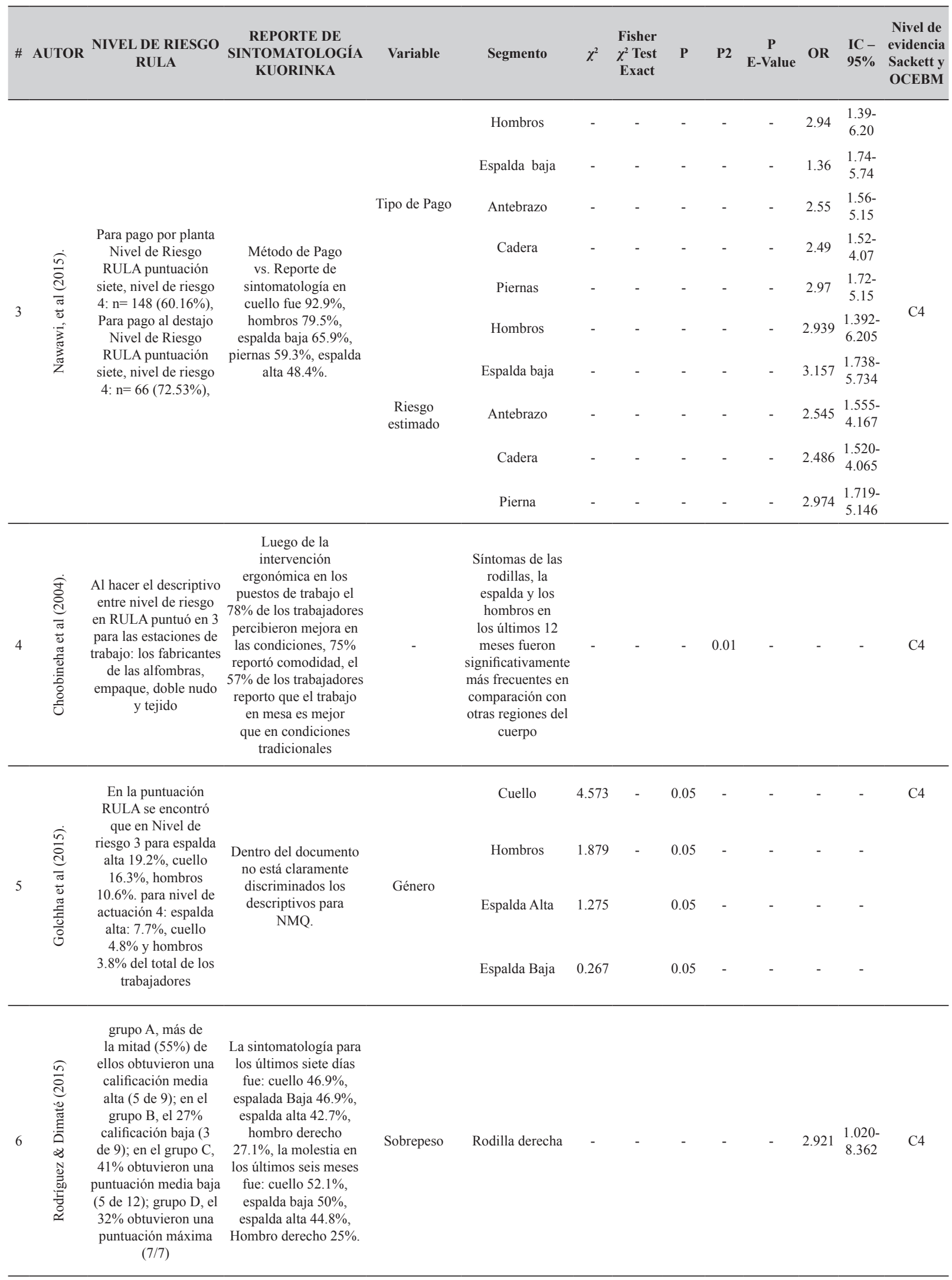


Salud UIS Vol.49 No.1 Enero - Marzo de 2017

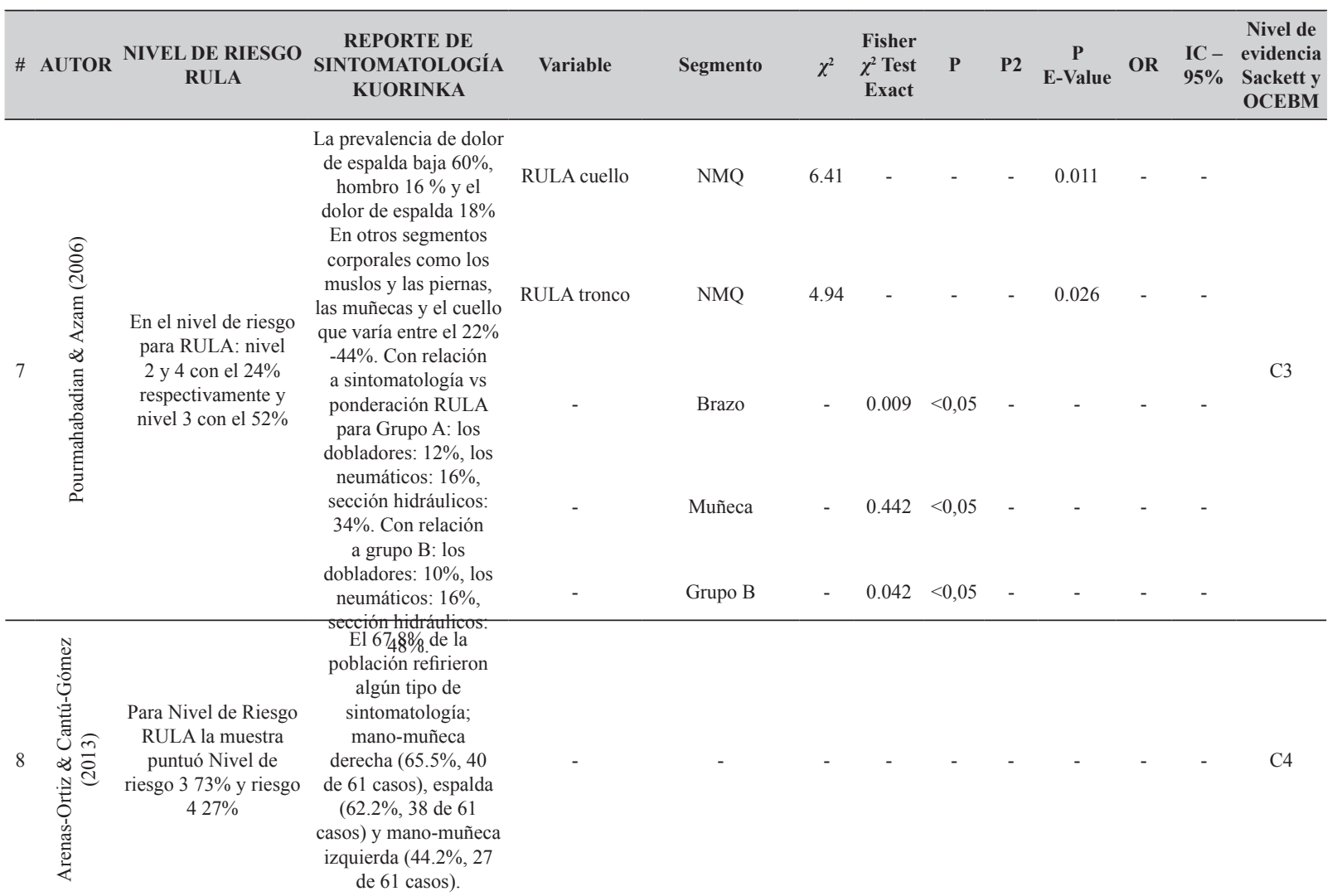

Trabajos para

regiones del excepto codos y cuerpo

El segmento corpora con mayor reporte

de sintomatología es espalda con $54.8 \%$, e segmento con menor reporte en muslos con $26.2 \%$. El envasado en forma de tabletas, la tasa de prevalencia de la parte superior de la espalda es mayor (19\%) que el resto de las operaciones de embalaje para las áreas

rabes, Ampoll

\begin{tabular}{|c|c|c|c|c|c|c|c|c|c|}
\hline & Cuello & 0.001 & - & - & $\begin{array}{l}18.55 \\
(4 \mathrm{df})\end{array}$ & - & - & - & \\
\hline & Hombros & 0.001 & - & - & $\begin{array}{l}18.66 \\
(4 d f)\end{array}$ & - & - & - & \\
\hline Segmentos & Mano Muñeca & 0.000 & - & - & $\begin{array}{l}21.92 \\
(4 \mathrm{df})\end{array}$ & - & - & - & \\
\hline $\begin{array}{l}\text { Corporales } \\
\text { Js. Reporte }\end{array}$ & Espalda Alta & 0.000 & - & - & $\begin{array}{l}24.67 \\
(4 \mathrm{df})\end{array}$ & - & - & - & \\
\hline $\begin{array}{l}\text { Diferentes } \\
\text { puestos de }\end{array}$ & Espalda Baja & 0.008 & - & - & $\begin{array}{l}13.90 \\
(4 \mathrm{df})\end{array}$ & - & - & - & \\
\hline trabajo & Muslos & 0.045 & - & - & $\begin{array}{l}9.75 \\
(4 d f)\end{array}$ & - & - & - & $\mathrm{C} 3$ \\
\hline & Rodillas & 0.012 & - & - & $\begin{array}{l}12.88 \\
(4 d f)\end{array}$ & - & - & - & \\
\hline & Piernas & 0.009 & - & - & $\begin{array}{l}13.42 \\
(4 \mathrm{df})\end{array}$ & - & - & - & \\
\hline & Cuello & 0.002 & 0.003 & - & $\begin{array}{l}9.80 \\
(1 \mathrm{df})\end{array}$ & - & - & - & \\
\hline & Hombros & 0.004 & 0.007 & - & $\begin{array}{l}8.34 \\
(1 \mathrm{df})\end{array}$ & - & - & - & \\
\hline \multirow[t]{3}{*}{$\begin{array}{l}\text { Sexo Vs } \\
\text { Reporte de } \\
\text { lor Embalaje }\end{array}$} & Codo & 0.01 & 0.01 & - & $\begin{array}{l}6.55 \\
(1 \mathrm{df})\end{array}$ & - & - & - & \\
\hline & Muslos & 0.02 & 0.02 & - & $\begin{array}{l}5.25 \\
(1 \mathrm{df})\end{array}$ & - & - & - & \\
\hline & Piernas & 0.001 & 0.002 & - & $\begin{array}{l}10.70 \\
(1 \mathrm{df})\end{array}$ & - & - & - & \\
\hline
\end{tabular}


Percepción de desórdenes musculoesqueléticos y aplicación del método RULA en diferentes sectores productivos: una revisión sistemática de la literatura

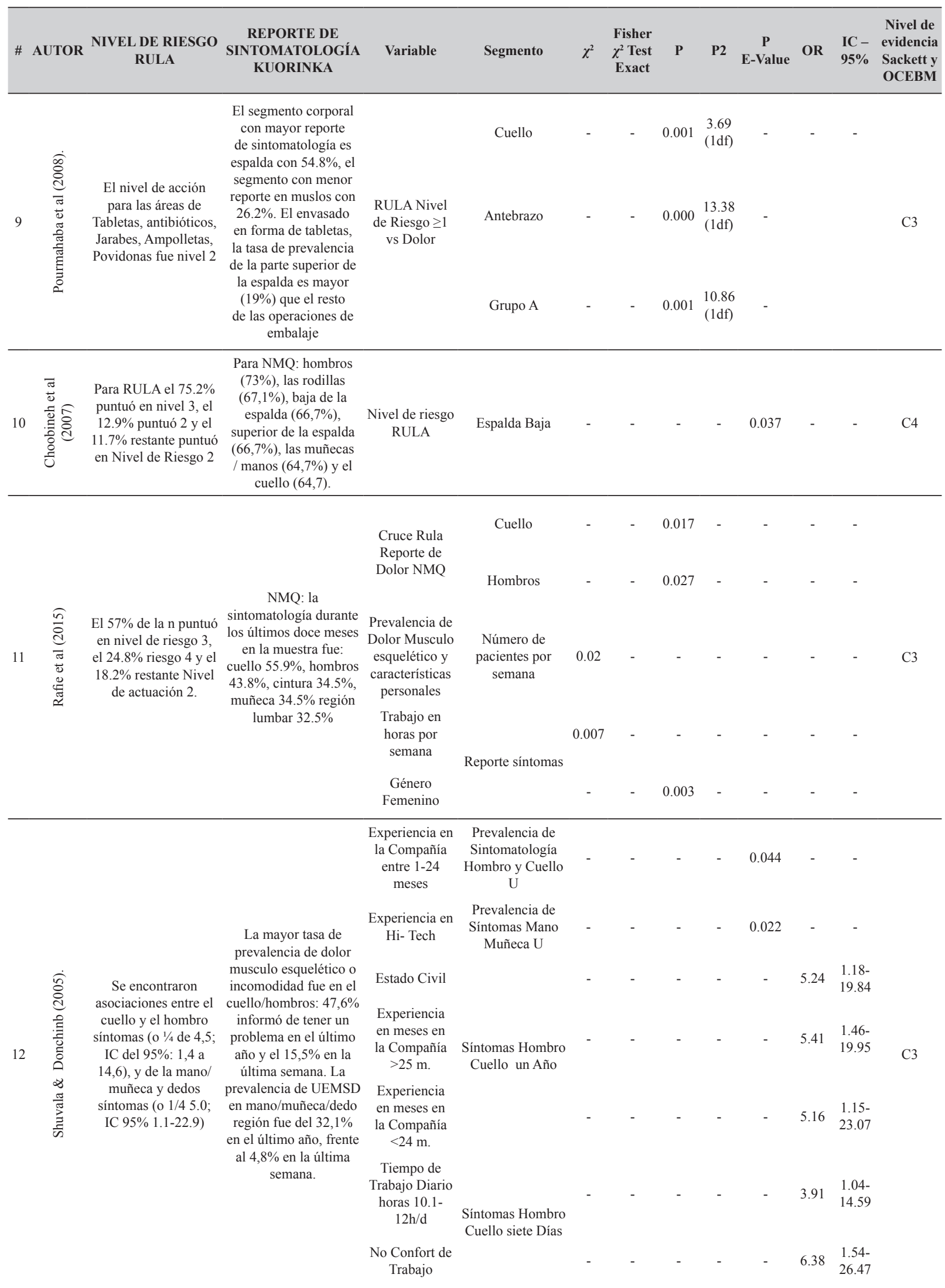


Salud UIS Vol.49 No.1 Enero - Marzo de 2017

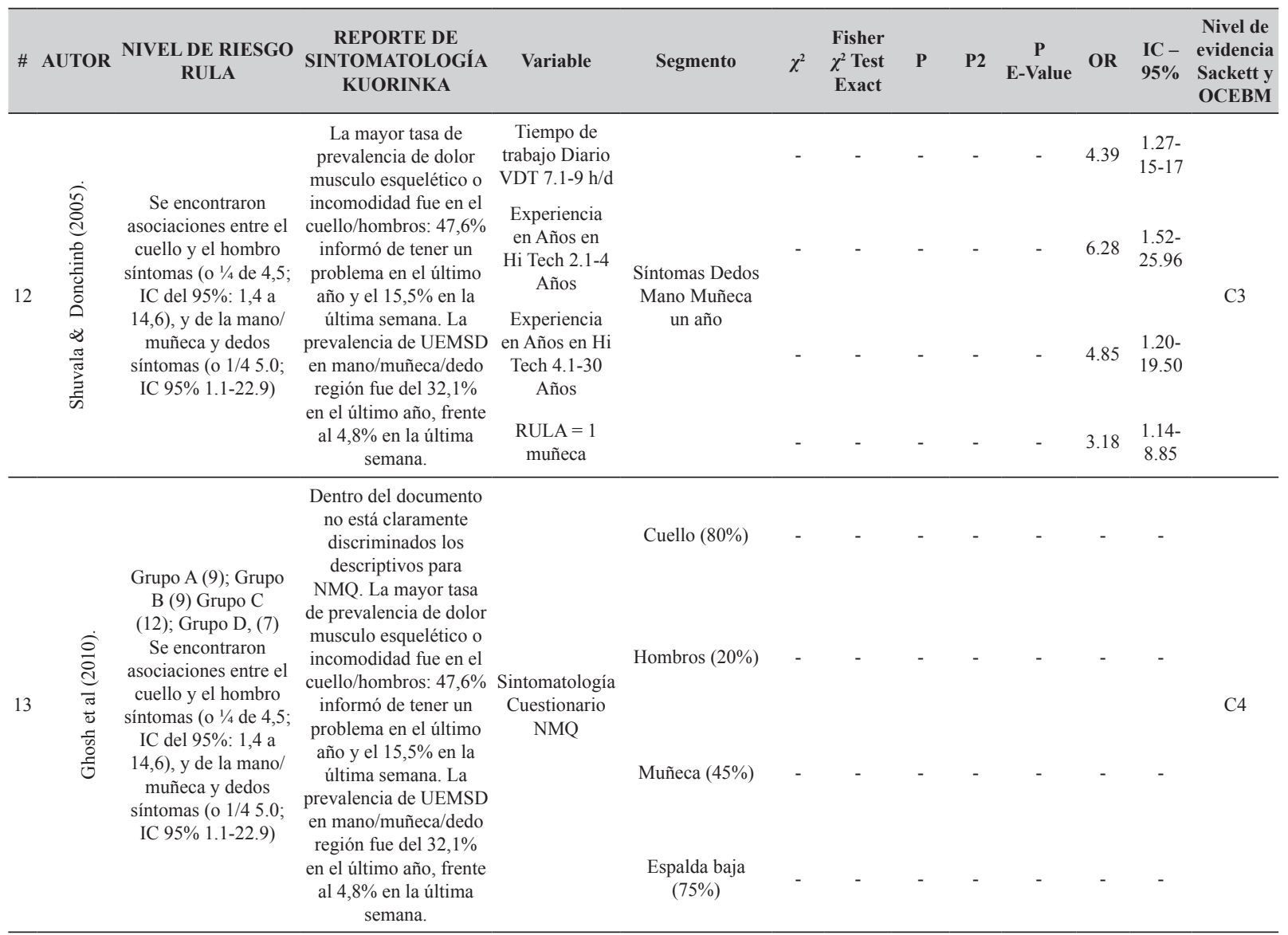

U: Univariado. M: Multivariado; NMQ: Cuestionario Nórdico

Fuente: Elaboración propia.

\section{DISCUSIÓN}

Los Principales hallazgos de la presente revisión sugieren que existe asociación entre el nivel de riesgo medio y alto arrojado por el método RULA para reporte de sintomatología en los trabajadores de los sectores económicos incluidos. Asimismo, se registró que en los segmentos donde la puntuación RULA es alta para grupo A (brazos y antebrazos) y zonas corporales del grupo B (cuello y tronco), hubo mayor reporte de sintomatología en los trabajadores de diversos sectores económicos.

\section{Grado de Riesgo Biomecánico con Método RULA en diversos sectores}

Al clasificar el grado de riesgo Biomecánico (RULA), se encontró en los trabajadores (operadores de máquina de coser en Malasia) que en su mayoría puntuaron en nivel 3, hubo asociación entre presencia de factores de riesgo ergonómico y método de pago de salario $(\text { destajo })^{35}$, resultados similares a los registrados en los operarios de una industria de alfombras en la primera

medición. Una vez se realizaron los ajustes sobre los puestos de trabajo la puntuación de RULA en dichas estaciones disminuyó a grado $2^{40}$.

De igual forma, grados altos de puntuación en el método RULA fueron encontrados en trabajadores de empaque de alimentos, nivel 3 y $4^{41}$, similar a los hallazgos encontrados en la industria automotriz ${ }^{38}$; lo anterior no difiere de los hallazgos en los operadores de máquina donde el nivel de riesgo fue alto (muñeca, cuello y tronco puntuaron 2 y 3 ) y puntuaciones globales de nivel 3 y 4; la media en la puntuación de Brazo y muñeca fue más alta en hombres (4.9) que en mujeres (4.5); sin embargo, la media para mujeres (4.1) en el Grupo B (cuello, tronco, piernas) fue más alta respecto a hombres (2.9). Además, se encontró una puntuación final media de 6,1 para mujeres y 5,4 para hombres ${ }^{39}$.

En operarios de prensa hidráulica (prensa de flexión y prensa de impacto), la puntuación final fue $2(52 \%)$, 3 y $4(42 \%)^{44}$, similar a lo obtenido en la industria farmacéutica con nivel 2 para todos los operadores de embalaje ${ }^{43}$; esto difiere de los hallazgos en trabajadores 
administrativos ${ }^{27} \mathrm{y}$ trabajadores de orfebrería en quienes el nivel de riesgo fue $4^{37}$.

Los hallazgos son similares a los encontrados en una compañía de comunicaciones en donde la puntuación final fue nivel 3 y $4^{42}$, resultados similares a los obtenidos en trabajadores de una empresa de alta tecnología en quienes la puntuación media en muñeca fue 2.3 , cuello 1.9 y la puntuación media final 4.5, lo cual indica que las posturas de los operarios en sus puestos de trabajo deben ser investigadas y modificadas. En el análisis de la variable sexo, las puntuaciones fueron similares en hombres y mujeres en brazo/muñeca ${ }^{34}$, lo que se asemeja a lo encontrado en un grupo de odontólogos donde las puntuaciones fueron altas 3 (57\%), muy altas $4(24.8 \%)$ e intermedias $2(18.2 \%)^{45}$, posiblemente por las demandas motrices de las tareas que implican carga postural estática.

\section{Asociaciones entre nivel de Riesgo Biomecánico y percepción de DME}

Según Dianat, et al. (2015) (operarios de máquina), los hombres reportaron mayor sintomatología en espalda alta mientras que la frecuencia de síntomas en mano/ muñeca fue mayor en mujeres; a su vez, se evidenció aumento de la sintomatología con la edad ${ }^{39}$, resultados similares fueron encontrados por Pourmahabadian, et al. (2008) (industria farmacéutica) con una asociación significativa entre el sexo (femenino) y el dolor (trabajadores de embalaje) en cuello, hombros, codo, muslos, rodillas y piernas ${ }^{43}$; lo anterior se contrapone a lo hallado por Shuval, et al. (empresa de alta tecnología), dado que la media del brazo/muñeca RULA fue idéntica en hombres y mujeres $(4,0)$; sin embargo, la puntuación fue mayor para mujeres en cuello/tronco/piernas (4.7 y 4.2 respectivamente, $\mathrm{p}=0,057)$ y en la puntuación final en RULA (media de 4.7 y 4.4 respectivamente, $p=0,132)^{34}$. Lo antes descrito, posiblemente se deba al tamaño de la muestra y la representatividad en la misma.

En lo concerniente al Índice de Masa Corporal (IMC), según Rodríguez, et al. (administrativos de una universidad), se encontró diferencias estadísticamente significativas entre IMC y sintomatología en la rodilla izquierda ${ }^{27}$, lo que es contrario a los hallazgos de Rafie, et al. (odontólogos ${ }^{45}$, posiblemente por diferencias en las variables sociodemográficas de edad y sexo, y en otras como actividad física.

En relación a las variables sociodemográficas, que son abordadas en todos los estudios revisados, escaza es la información que contiene asociaciones estadísticas.
No obstante, en el estudio realizado por Abd Rahman, et al. (Industria automotriz), se encontraron tres factores significativamente asociados con DME: edad, antigüedad y puntuaciones altas de RULA ${ }^{38}$. Por otra parte, en el estudio de Nawawi, et al. (operarios de máquina de coser), se encontró que trabajar por destajo tiene asociación con la aparición de DME en hombro, espalda baja, antebrazo, cadera y pierna ${ }^{35}$; asimismo, Rafie, et al. (odontólogos) observaron relaciones significativas entre DME y horas de trabajo diario y el número de pacientes ${ }^{45}$.

Si bien, en los estudios incluidos en la presente revisión se hizo uso del método RULA y Cuestionario Nórdico, solo en el $46 \%$ de ellos se hallaron asociaciones estadísticamente significativas (pese a los diseños metodológicos). Lo anterior es el caso del estudio realizado en trabajadores de prensa hidráulica donde hubo asociación significativa entre RULA y el dolor en cuello, tronco, brazos y zonas corporales del grupo $\mathrm{B}^{44}$, similar a lo encontrado en odontólogos, en quienes se identificaron asociaciones entre RULA y síntomas en cuello y hombros ${ }^{45}$, y entre el nivel de riesgo y DME en espalda baja (compañía de comunicaciones) ${ }^{42}$; aunado a lo anterior, en la industria farmacéutica se estableció asociación entre la puntuación 1 (RULA) por segmento y reporte de síntomas en cuello, antebrazo y grupo A, además de encontrarse relación estadística entre RULA $(\geq 1)$ y molestia en cuello, antebrazo, muñeca/manos, y reportándose también asociación entre dolor y nivel de riesgo en las regiones del cuerpo, excepto para codos y tobillo ${ }^{43}$.

Paralelamente, en la Industria automotriz se identificó asociación entre trabajadores calificados con nivel 3 y 4 (RULA) y $\mathrm{DME}^{38}$, resultados relativamente altos en relación con otras industrias. Sin embargo, en el estudio de Nawawi, et al. al comparar la puntuación final de RULA con la aparición de DME en operadores de máquina, se encontró asociación estadísticamente significativa para hombro, espalda baja, antebrazo, cadera y pierna ${ }^{35}$. Estos resultados, posiblemente están dados por los ciclos de trabajo y la repetitividad en las tareas en las líneas de trabajo analizadas para los dos sectores.

La revisión sugirió que el uso del método RULA y Cuestionario Nórdico pueden tener una relación y son susceptibles de ser usadas para la evaluación de los DME de origen laboral. La propuesta de enfoque integrador puede ayudar en el desarrollo de estrategias de intervención para los DME de origen laboral, en el que RULA puede utilizarse como una herramienta de tamizaje para riesgo postural siguiendo una breve 
sesión de entrenamiento, independientemente de la experiencia del asesor en las evaluaciones de riesgo postural $^{36}$.

Las principales limitaciones de la presente revisión son la no inclusión de artículos publicados en otros idiomas diferentes al inglés, español y portugués, y el uso del material que se encontró de acceso libre y con el texto completo. Cabe mencionar que una de las limitantes que tiene el método RULA es que evalúa la peor postura registrada durante el trabajo habitual, por lo que no se tiene en cuenta la carga total durante los diferentes ciclos de las tareas. Otra de las limitantes en términos del análisis radicó en la poca homogeneidad en el análisis estadístico realizado en los estudios, debido al tamaño de la muestra, muestreo y otras variables, entre ellas, sociodemográficas y organizacionales.

\section{CONCLUSIONES}

La revisión sistemática muestra el limitado uso del método RULA para la detección del grado de riesgo biomecánico en conjunto con la aplicación del Cuestionario Nórdico (percepción de los DME) en diversos sectores económicos; sin embargo, los resultados sugieren que hay nivel de riesgo alto o muy alto identificado con RULA (grado 3 o 4) y presencia de DME en trabajadores de la industria automotriz, prensa hidráulica, odontólogos, administrativos, operarios de empaque de alimentos, máquina de coser, industria farmacéutica, alta tecnología e industria manufacturera (como orfebres y tejedores de alfombras), existiendo una situación de relativa desventaja para el género femenino.

Se encontraron prevalencias altas de DME en espalda alta (industria farmacéutica, empresa de alta tecnología, ensambladores de la industria automotriz y tejedores de alfombras), espalda baja (ensambladores de la industria automotriz, industria de comunicaciones y odontólogos), cuello, hombros, la región de mano/ muñeca (operarios de máquina de coser, orfebres, operadores de máquinas, empresa de alta tecnología, administrativos de una universidad, industria de empaquetado de frijol y odontólogos) y rodilla, siendo el género femenino el que presenta mayor presencia de sintomatología.

Los trabajadores de la industria manufacturera muestran una percepción adversa de su salud influenciada por la presencia de posturas inadecuadas y carga estática elevada; asimismo, se puede sugerir asociación entre índice de masa corporal (administrativos), edad mayor a
25 años (industria automotriz), antigüedad en el trabajo ( $\geq 3$ años) (industria automotriz), trabajo por destajo (operarios de máquina de coser), horas de trabajo diario $\geq 8$ horas (odontólogos), número de pacientes atendidos (odontólogos) y sintomatología. Aunado a lo anterior, hubo asociación entre RULA (nivel 3 y 4) y dolor reportado en: cuello, tronco, brazos, antebrazos, zonas corporales del grupo B (trabajadores de prensa hidráulica, odontólogos e industria farmacéutica) y espalda baja (industria automotriz, compañía de comunicaciones y operadores de máquinas de coser). No obstante, debido al diseño metodológico de algunos estudios no es concluyente la relación entre el grado de riesgo biomecánico y la sintomatología.

Así pues, hay datos de calidad insuficiente para recomendar el uso frecuente del método RULA en combinación con el Cuestionario Nórdico de Kuorinka para la evaluación de tareas las cuales demanden carga combinado. A pesar de ello, estos dos métodos pueden ser buenos predictores de aparición de DME en presencia de carga estática. Según la presente esta revisión sistemática, la aplicabilidad de los dos instrumentos se encuentra limitada debido al sesgo de publicación y las limitaciones del diseño de estudio de los documentos individuales hallados; por lo anterior, se recomienda desarrollar estudios de mayor grado de evidencia para poder establecer la efectividad de los métodos aplicados en conjunto.

\section{CONFLICTO DE INTERÉS}

Los autores declaran ningún conflicto de interés.

\section{AGRADECIMIENTOS}

Esta investigación fue apoyada por la Maestría en Salud Pública de la Fundación Universitaria del Área Andina y la Universidad Nacional de Colombia (Bases de datos).

\section{REFERENCIAS}

1. Kanchanomai S, Janwantanakul P, Pensri P, Jiamjarasrangsi W. Prevalence of and factors associated with musculoskeletal symptoms in the spine attributed to computer use in undergraduate students. Work. 2012; 43(4): 497-506. DOI: 10.3233/WOR-2012-1387.

2. Ministerio de la Protección Social. Guias de Atención Basadas en la Evidencia (Gatiso) Desórdenes Musculo Esqueleticos DME Relacionados con los movimientos repetitivos de Miembros Superiores. Documento Técnico. Bogotá: Ministerio de la 
Protección Social; 2007. Report No.: 978-95898067-4-6.

3. Organización Mundial de la Salud. Prevención de trastornos musculoesqueléticos en el lugar de trabajo. Serie protección de la salud trabajadores \# 5. Diocumento Técnico. Ginebra: Organización Mundial de la Salud (OMS); 2004. Report No.: ISBN:9243591002.

4. Organización Mundial de la Salud. Crear Lugares de Trabajo Saludables y Equitativos para Hombres y mujeres. Documento. Ginebra: Organización Mundial de la Salud (OMS); 2011. Report No.: ISBN: 9789243501734.

5. Asensio-Cuesta, Sabina MJ, Bastante Ceca JAD. Evaluación ergonómica de puestos de trabajo. 1st ed. Madrid: Editorial Paraninfo; 2012.

6. Díaz C, de Vicente A, Zimmermann M, Galiana L. El trastorno musculo-esquelético en el ámbito laboral en cifras. Documento técnico. Madrid: Ministerio de Empleo y Seguridad Social España; 2012. Report No.: NIPO272-13-027-7.

7. Bhattacharya A. Costs of occupational musculoskeletal disorders (MSDs) in the United States. Int J Ind Ergon. 2014; 44(3): 448 - 454. DOI: http://dx.doi.org/10.1016/j.ergon.2014.01.008.

8. Bernard BP. Musculoskeletal disorders and workplace factors: a critical review of epidemiologic evidence for work-related musculoskeletal disorders of the neck, upper extremity, and low back. Documento. Cincinnati: U.S. Department of Health and Human Services; 1997. Report No.: Publicación 97B141.

9. Health and Safety Executive. Health and safety statistics Annual Report for Great Britain. Informe Estadisticas. Health and Safety Executive; 2015.

10. Doulliet P, Aptel M. Prevencion de los Trastornos Musculo esqueléticos: hacia un planteamiento Global. magazin. 2001.

11. Janwantanakul P, Pensri P, Jiamjarasranqsi W, Sinsongsook T. The relationship between upper extremity musculoskeletal symptoms attributed to work and risk factors in office workers. Int Arch Occup Environ Health. 2010; 83(3); 273-281. DOI: 10.1007/s00420-009-0466-x.

12. Riihimäki H, Viikari-Juntura E. Enciclopedia salud y seguridad en el trabajo Sociales MdTyA, editor. Madrid: Chantal Dufresne, BA; 1998.

13. Cañas JJ, Waerns Y. Ergonomía cognitiva: aspectos psicológicos de la interacción de las personas con la tecnología de la información.: Ed. Medica Panamericana; 2001.

14. Romero JCR. Métodos de evaluación de riesgos laborales Trabajo INdHySee, editor. Madrid:
Ediciones Díaz de Santos; 2004.

15. Corlett EN, Madeley SJ, Manenica I. Posture targeting: a technique for recording working postures. Ergonomics. 1979; 22(3): 357-366. DOI: http://dx.doi.org/10.1080/00140137908924619

16. Mondelo PR, Torada EG, Bombardo PB. Fundamentos de Ergonomía; Universidad Politécnica de Catalunya. Universitat Politecnica de Catalunya ed. Catalunya: Mutua Universal; 2010.

17. Universidad Politécnica de Valencia. Evaluación Postural Rápida EPR. 2006.

18. Occhipinti E. OCRA: a concise index for the assessment of exposure to repetitive movements of the upper limbs. Ergonomics. 1998; 41(9): 12901311.

19. Schnall PL, Pieper C, Schwartz JE, Karasek A, Schlussel , Devereux B, et al. The relationship between 'job strain,' workplace diastolic blood pressure, and left ventricular mass index. JAMA. 1992; 263(14): 1929-1935.

20. McAtamney L, Corlett EN. RULA: a survey method for the investigation of work-related upper limb disorders. Appl Ergon. 1993; 24(2): 91-99.

21. Kuorinka I, Jonsson B, Kilbom A, Vinterberg $\mathrm{H}$, BieringSorensen F, Andersson G, et al. Standardised nordic questionnaires for the analysis of musculoskeletal symptoms. Appl Ergon. 1887; 18(3): 233-237. DOI: http://dx.doi. org/10.1016/0003-6870(87)90010-X.

22. Kuorinka I, Forcier L. Work Related Musculoskeletal Disorders (WRMDs): A Reference Book for Prevention. Taylor \& Francis. 1995.

23. Dawson P, Steele J, Hodges PW, Stewart. Development and test-retest reliability of an extended version of the Nordic Musculoskeletal Questionnaire (NMQ-E): a screening instrument for musculoskeletal pain. J Pain. 2009; 10(5): 517-526. DOI: 10.1016/j.jpain.2008.11.008.

24. Crawford JO. The Nordic musculoskeletal questionnaire. Occup Med. 2007; 57(4): 300-301. DOI: https://doi.org/10.1093/occmed/kqm036.

25. Vernaza Rincón P, Sierra Torres C. Dolor Musculo esquelético y su asociación con factores de riesgo ergonómicos, en trabajadores administrativos. Rev Sal Pública. 2005; 7(3): 317-326.

26. Cook C, Burgess-Limerick R, Chang S. The prevalence of neck and upper extremity musculoskeletal symptoms in computer mouse users. Int J Ind Ergon. 2000; 26(3): p. 347-356. DOI: http://dx.doi.org/10.1016/S0169-8141(00)00010-X.

27. Rodríguez-Romero D, Dimaté-García AE. Evaluación de riesgo biomecánico y percepción de desórdenes músculo esqueléticos en administrativos 
de una universidad Bogotá (Colombia). Inv Andina. 2015; 17(31): 1284-1299.

28. Mishra W, Amitabha D, Iqbal R, Gangopadhyay S, Chandra AM. An integrative approach for evaluating work related musculoskeletal disorders. Work. 2012; 43(4): 437-446. DOI: 10.3233/WOR2012-1460.

29. Moher D, Liberati A, Tetzlaff J, Altman DG, Prisma Group. Preferred reporting items for systematic reviews and meta-analyses: the PRISMA statement. BMJ. 2009; 339: B2535. DOI: 10.1136/bmj.b2535.

30. Hernandez Sampieri R, CFCMdpBL. Metodologia de la Investigación: McGrawHill; 2010.

31. Beltrán OA. Revisiones sistemáticas de la literatura. Rincón Epidemiol. 2005; 20(1): 60-69.

32. Ferreira González I, Urrutia G, Alonso-Coello P. Revisiones sistemáticas y metaanálisis: bases conceptuales e interpretación. Rev Esp Cardiol. 2011; 64(8): 688-696. DOI: http://dx.doi. org/10.1016/j.recesp.2011.03.029.

33. McAtamney L, Nigel CE. RULA: a survey method for the investigation of work-related upper limb disorders. Appl Ergon. 1993; 24(2): 91-99.

34. Shuval K, Donchin M. Prevalence of upper extremity musculoskeletal symptoms and ergonomic risk factors at a Hi-Tech company in Israel. Rev Int Ergon Ind. 2005; 35(6): 569-581. DOI: http://dx.doi. org/10.1016/j.ergon.2005.01.004.

35. Nawawi R, Deros B, Daruis D, Ramli A, Zayn R, José L. Effects of payment method on work control, work risk and work-related musculoskeletal health among sewing machine operators. Journal of Mechanical Engineering and Sciences. 2015; 9: p. 1705-1713. DOI: 10.15282/jmes.9.2015.16.0164.

36. Golchha , Sharma , Wadhwa , Yadav, Paul. Ergonomic risk factors and their association with musculoskeletal disorders among Indian dentist: a preliminary study using Rapid Upper Limb Assessment. Indian J Dent Res. 2014; 25(6): 767771.

37. Ghosh T, Das B, Gangopadhyay S. Work-related musculoskeletal disorder: an occupational disorder of the goldsmiths in India. Ind $\mathrm{J}$ Community Med. 2010; 35(2): 321-325. DOI: 10.4103/09700218.66890.

38. Abd Rahman A, Yazdani A, Kadir Shahar H, Adon MY. Association between awkward posture and musculoskeletal disorders (MSD) among assembly line workers in an automotive industry. Malaysian J Med Health Sci. 2014; 10(1): 22-28.

39. Dianat I, Kord M, Yahyazade P, Karimi MA, Stedmon AW. Association of individual and workrelated risk factors with musculoskeletal symptoms among Iranian sewing machine operators. Appl Ergon. 2015; 51: 180-188. DOI: 10.1016/j. apergo.2015.04.017.

40. Choobineh, Tosian R, Alhamdi, Davarzanie. Ergonomic intervention in carpet mending operation. Appl Ergon. 2004; 35(5): 493-496.

41. Arenas-Ortiz L, Cantú-Gómez Ó. Factores de riesgo de trastornos músculo-esqueléticos crónicos laborales. Med Int Méx. 2013; 29(4): 370-379.

42. Choobineh A, Tabatabaei SH, Tozihian M, Ghadami F. Musculoskeletal problems among workers of an Iranian communication company. Indian J Occup Environ Med. 2007; 11(1): 32-36. DOI: 10.4103/0019-5278.32462.

43. Pourmahabadian M, Akhavan M, Azam K. Investigation of risk factors of work-related upperlimb musculoskeletal disorders in a pharmaceutical industry. J Appl Sci. 2008; 8(7): 1262-1267.

44. Pourmahabadian M, Azam K. Evaluation of risk factors associated with work-related musculoskeletal disorders of upper limbs extremity among press workers. Pak J Med Sci. 2006; 22(4): 379-384.

45. Forouzan R, Zamani Jam A, Shahravan A, Raoof M, Eskandarizadeh A. Prevalence of upper extremity musculoskeletal disorders in dentists: symptoms and risk factors. J Environ Public health. 2015; 2015. DOI: http://dx.doi.org/10.1155/2015/517346. 